\title{
Review of Clinical Manifestations and Impact of Parasitic Protozoan Infections on Human Reproductive Health
}

\author{
Mulambalah Chrispinus Siteti ${ }^{1,}$,, Siteti Darwin Injete ${ }^{2}$ \\ ${ }^{1}$ Department of Medical Microbiology \& Parasitology, School of Medicine, College of Health Sciences, Moi University, Eldoret, Kenya \\ ${ }^{2}$ College of Health Sciences, Jomo Kenyatta University of Agriculture and Technology, Nairobi, Kenya
}

Email address:

csmulambalah@gmail.com (M. C. Siteti), darwinsiteti@gmail.com (S. D. Injete)

\section{To cite this article:}

Mulambalah Chrispinus Siteti, Siteti Darwin Injete. Review of Clinical Manifestations and Impact of Parasitic Protozoan Infections on Human Reproductive Health. Clinical Medicine Research. Vol. 4, No. 2, 2015, pp. 27-33. doi: 10.11648/j.cmr.20150402.11

\begin{abstract}
Parasitic protozoan infections that affect reproductive health are being increasingly recognized as a serious global health problem with impact on individual women and men, their families and communities. The infections can have severe consequences, including infertility, ectopic pregnancy, chronic pelvic pain, miscarriage, and increased risk of HIV transmission. Some affect both men and women, and can also be transmitted from mothers to children during pregnancy and childbirth. These infections affect more than just reproductive health, the resultant morbidity also affects the economic productivity and quality of life of many individual women and men, and consequently, of whole communities, regions and countries. The review intents to re-focus the attention of health professionals on this subject by shedding light on these unjustly neglected parasitoses commonly considered of low public health importance. Many of these parasitic infections have been forgotten and have become the neglected diseases often overlooked and rarely a high priority globally.
\end{abstract}

Keywords: Protozoal Infections, Reproductive Health, Pregnancy Outcomes, Infertility

\section{Introduction}

World Health Organization defines health as a state of complete physical, mental and social well-being, and not merely the absence of disease or infirmity. Reproductive health [also known as sexual health] is a subset of health and addresses the reproductive processes, functions and systems at all stages of life [1]. Reproductive health implies that people are able to have a responsible, satisfying and safer sex life and that they have the capability to reproduce. The definition implies that men and women ought to be informed of access to appropriate health care services of sexual, reproductive medicine and implementation of health education programs. It also emphasizes the importance of women to go safely through pregnancy and childbirth and the need to provide couples with the best chance of having a healthy infant.

Low income individuals in many developing countries lack the resources for appropriate health services and the knowledge on what is appropriate for maintaining acceptable reproductive health [2]. This is because of lack of knowledge on reproductive health and associated diseases and yet reproductive health is considered as a part of sexual and reproductive health rights. According to the world health organization, reproductive and sexual ill-health accounts for $20 \%$ of the global burden of ill-health for women and $14 \%$ for men [1].

Infectious diseases are known to affect reproductive health either directly or indirectly causing approximately $60 \%$ morbidity. In this regard, a number of species of pathogenic protozoa that in many cases do not localize in the reproductive system are implicated. A variety of protozoal infections in humans which are not usually sexually transmitted show symptoms that mimic classic sexually transmitted infections by producing ulcerations for instance amoebiasis, leishmaniasis or lesions of the upper genital tract for instance salpingitis as a result of amoebiasis.

Parasite adverse effects in reproductive health differ depending on the sex of the individual and clinical presentation varies and may mimic and be confused with other unrelated clinical conditions. Generally the manifestations include genital deformation, inflammation, infertility, spontaneous abortion, still birth and enhancing the likelihood of HIV co-infection. Typically many adolescent girls and women exhibit several symptoms in their lower genital tract where overt bleeding, unpleasant discharge, general discomfort and pain during sex can lead to low self- 
esteem, depression and stigma and death.

There are several reasons why we should focus more attention to any factor or aspect that negatively impacts on human reproductive health in either gender. Reproductive health is a crucial part of general health and a central feature of human development. It is considered as a reflection of health during childhood and crucial during adolescence and adulthood, sets the stage for health beyond the reproductive years for both women and men. It may consequently affect the health of the next generation because the health of the newborn is largely a function of the mother's health status and of her access to health care [3] specifically reproductive healthcare.

Reproductive health is not only a universal concern but is of special importance for women particularly during the reproductive years. Although most reproductive health problems arise during the reproductive years, it is noted that old age general health is a reflection of earlier reproductive life events during which many infections are acquired. Men too have reproductive health concerns and needs though their reproductive health is affected to a lesser extent than is the case for women. However, men have special roles and responsibilities (as head of family) in terms of women's reproductive health. This is due to their decision-making powers in reproductive health matters at family level. In this regard, there are several reasons why involving men in reproductive health studies and programs is important. Studies show that when men are provided with information about reproductive health issues, they are likely to be increasingly influential on their partner's family planning decisions and treatment seeking behavior [4].

Every individual male or female has different needs and problems at every stage in life that relate to reproductive health that have important implications for future well-being. Failure to deal with reproductive health problems at any stage in life sets the scene for later health and developmental problems [5]. It is also a social and economic imperative because human energy and creativity are the driving forces of development. Such energy and creativity cannot be generated by sick, tired people, and consequently a healthy and active population becomes a prerequisite of social and economic development [3].

This review addresses specific parasitic infections that cause significant morbidity that affect directly or indirectly human reproductive health namely, trichomoniasis, amoebiasis, leishmaniasis, toxoplasmosis and malaria individually. We highlight the mode of infection, clinical manifestations, risk of other infections, genital and pregnancy complications/outcomes and impact on reproductive health in males and females. The review intents to re-focus the attention of the health professionals on this subject by shedding light on these unjustly neglected parasitoses commonly considered of low public health importance. Many of these parasitic infections have been forgotten and have become the neglected diseases often overlooked and rarely a high priority globally.

\section{Protozoan Infections and Reproductive Health}

Protozoan species from several genera are commonly associated with infections prevalent in reproductive age of women and men with serious morbidity reproductive wastage. These are the genus Trichomonas, Plasmodium, Entomoeba, Leishmania and Toxoplasma. Parasite species in the genera are variously associated with vaginitis, endometritis, adnexitis, pyosalpinx, infertility, preterm birth, low birth weight, bacterial vaginosis, and increased risk of cervical cancer, human papilloma virus (HPV), and human immunodeficiency virus (HIV) infections in women. In men, complications include urethritis, prostatitis, epididymitis, and infertility through inflammatory damage or interference with the sperm motility and function. Only Trichomonas localizes in the urinogenital system and therefore directly affects the reproductive system causing venereal disease, trichomoniasis. Species in the other four genera cause multi-systemic diseases that have serious implications on reproductive health.

\subsection{Human Trichomoniasis}

Human trichomoniasis is caused by Trichomons vaginalis, a sexually transmitted extracellular flagellated single-cell parasite that lives in the female lower reproductive tract and the urethra in males. $T$. vaginalis selectively adheres to the human vaginal epithelial cells. The parasite has been extensively studied, but still little is known about the immunopathogenesis of trichomoniasis and the molecular mechanisms exploited to evade the immune system. A unique lipophosphoglycan glycosyl-phosphatidylinositol (GPI) molecule is widely reported to play a major pathogenic and immunoregulatory role $[6,7,8,9,10]$. The parasite is also reported to carry and disseminate viruses and Mycoplasma that are linked to the severity of mucosal damage, inflammatory symptoms, and consequences for reproductive outcome [11].

In females, $T$. vaginalis causes a wide spectrum of symptoms, ranging from a relatively asymptomatic state to severe inflammation and irritation with foul-smelling vaginal discharge, lower abdominal pain and dysuria. The associated morbidities and complications include vaginitis, endometritis, adnexitis, pyosalpinx, atypical pelvic inflammatory disease, preterm birth, premature membrane rupture, low birth weight, infertility, cervical cancer, enhanced risk of HIV-1 and other viral infections, and a positive association with bacterial vaginosis $[12,13]$. In isolated cases female infants become infected during birth and such infections may remain asymptomatic until puberty [14]. Trichomoniasis in men is usually self-limiting and often asymptomatic, it is associated with urethritis, prostatitis, epididymitis, reduced sperm function, and infertility $[9,15,16]$.

Worldwide 160 million to 180 million people are infected with trichomoniasis each year, and of those 154 million are in developing resource-limited countries and 8 to 10 million in developed countries. These may be underestimates [17] 
because the diagnostic tests used may not be confirmatory and also, results may not be representative of the general population, as the infection is asymptomatic in at least onethird of women and most men. An alarming high prevalence of trichomoniasis has been reported in healthy asymptomatic women attending family planning clinics in Africa [18] and $47 \%$ in the general population attending obstetrics and gynecology clinics in Asia [19]. In this regard, human trichomoniasis is considered as one of the most common non-viral sexually transmitted infection that has reached global epidemic level thus raising worldwide concern. The public health importance of the problem is underscored by the fact that the trichomoniasis infection is more prevalent in women /men of reproductive age with serious adverse reproductive outcomes [12]. The infection is on the rise in adolescents [20, 21] worldwide.

The parasite is known to produce proteases, mediators and intermediates associated with immunoinflammatory response to in pregnant women. These include alpha-defensin reported in pregnant infected women [22] and soluble leukocyte protease inhibitor (SLPI) and reactive nitrogen intermediates [23]. These and the presence of increased C-reactive protein in the sera of trichomoniasis infected pregnant women suggests that the impact of the immunoinflammatory reaction to the parasite exceeds the boundaries of the reproductive tract mucosa and may result in some of the pregnancy complications [22].

Many of the mechanisms of pregnancy complications linked to $T$. vaginalis remain elusive. The complex interactions between the parasite, the vaginal microflora and the host may hold the key to these complications and need to be addressed by future wider and long term clinical studies. Such studies should focus on the recently discovered unique proteins, the galectins, that represent an evolutionarily conserved family of glycoproteins that are essential for parasite survival, enhances virus attachment to cells and possibly modulates virulence and pathogenicity.

Further studies are also required to identify the host receptors and the host's as well as parasite's pathways involved in the symptomatic and asymptomatic sequelae and immune evasion by Trichomonas vaginalis. The mechanisms of the synergistic interactions between $T$. vaginalis and bacterial vaginosis, the most common vaginal syndrome of reproductive age women, are virtually unknown. More research is needed to understand and possibly prevent the negative impact of trichomoniasis on women's reproductive health and the risk of other sexually transmitted viral and bacterial diseases.

\subsection{Genital and Congenital Toxoplasmosis}

Toxoplasmosis is caused by a coccidian parasite Toxoplasma gondii, an intracellular protozoan that infect approximately one-third of the world's population. Many warm-blooded animals including the domestic cat act as definitive host whereas humans act as an intermediate hosts. The infection in humans generally occurs through consuming food or drink contaminated with developmental stages, oocysts or tissue cysts. Congenital transmission and organ transplantation are the other common routes of the infection [24]. Human toxoplasmosis is spreading fast in many different parts of the world [25] with sero-prevalence rate estimated between $20-80 \%$ thus raising global concern. The most common form of the infection in humans is latent (asymptomatic) infection but in some conditions like immune-compromised patients and congenitally infected fetuses and newborns, the parasite is reported to cause much more devastating and severe disease [26, 27].

After ingestion of parasite and proliferation of tachyzoites during acute stage, the parasite usually localizes in different organs and tissues [28, 29] including male and female reproductive organs of intermediate hosts including humans $[30,31,32,33,34,35,36]$. In these sites, the infection is associated with some adverse effects on the reproductive functions. In the recent years profound adverse effects of Toxoplasmosis infection on female reproductive functions have been reported [37] however, reports on male reproductive parameters have been rare but several findings are emerging in literature.

Clinical studies indicate that toxoplasmosis is associated with male sterility. In this regard [38] it has been reported that, infection with $T$. gondii in infertile couples is significantly higher than fertile couples. The study confirmed that the level of anti-sperm antibody was significantly higher in Toxoplasma infected than non-infected couples. In a related study in China, infertile cases of man's sterility, 36\% were associated with toxoplasmosis. Furthermore, several reports express association of male genital tract impairment with testicular toxoplasmosis [30, 31, 32, 33, 34, 35] and hypogonadotropic hypogonadism caused by congenital toxoplasmosis [36]. It is evident that toxoplasmosis infection is likely to become a major cause of male sterility and infertility worldwide in the near future.

Toxoplasma gondii may also be transmitted during pregnancy to the foetus in immunocompetent and immunocompromised women and result in congenital toxoplasmosis. The mechanism of vertical transmission is not yet understood. A probable scenario is that temporary parasitaemia in primarily infected pregnant women may result in invasion of the placenta by developmental stages known as tachyzoites which then multiply within cells of the placenta. Eventually, some of these may cross the placenta and enter the foetal circulation and subsequently foetal tissues $[39,40]$. Congenital toxoplasmosis is widely reported to cause abortion, neonatal death, or foetal abnormalities with detrimental consequences for the foetus [39, 41, 42, 43]. The infection significantly reduces the quality of life in children who survive a prenatal infection as cases of brain damage in new borne are increasingly attributed to cerebral toxoplamosis.

The incidence of prenatal infection with $T$. gondii is estimated to vary from 1 to 100 per 10000 births in different countries [39]. The risk of intrauterine infection of the foetus, the risk of manifestation of congenital toxoplasmosis, and the severity of the disease depend on several factors. These 
include: the time of maternal infection during pregnancy, the immunological competence of the mother during parasitaemia, the number and virulence of the parasite strain transmitted to the foetus, and the age of the foetus at the time of transmission. If not treated, the risk of intrauterine infection of the foetus increases during pregnancy. There is usually low risk during primary maternal infection in the first trimester and higher risk after primary maternal infection in the last trimester $[39,42]$. Because of this, the incidence of prenatal infection with $T$. gondii varies from the incidence of primary maternal infection during pregnancy. Toxoplasmosis is therefore an important infectious disease of animals and humans that may result in abortion, stillbirth or foetuses born with congenital infection hence result in reproductive wastage.

\subsection{Genital Amoebiasis}

Amoebiasis is caused by a protozoan Entamoeba histolytica. The classic effects of infection with Entamoeba histolytica are the development of a colitis that gives rise to amoebic dysentery and liver abscess when organisms enter the portal system [44]. Entamoba hitolytica has also been reported as a cause of genital ulcers. Possible modes of transmission of the parasite to genitourinary tract include hematogenous route, contiguous spread through perianal skin, oral/anal sex. In a review of 148 case reports on genital amoebiasis, $85 \%$ of reports confirm that genital infection in females, including infants is common [45] and this is becoming a worldwide concern.

Female genital amoebiasis is characterized by a foul, bloody vaginal discharge, abdominal pain, genital ulceration, often mimicking carcinoma of cervix. In a number of cases genital amoebiasis and carcinoma have been found together [46]. Genital amoebiasis involving the uterus [47] and Fallopian tubes [48] has also been reported. Most of the cases have diagnosed by cervical cytology and a few by ulcer biopsy. Most genital amoebiasis cases $(86 \%)$ in men present as a painful, discharging ulcers often mimicking carcinoma as in females and a few (14\%) with discharge or dysuria. Genital amoebiasis lesions generally respond swiftly to a standard course of metronidazole treatment. However, neglected cases in female patients progress to bloody discharge, small ulcerated and friable lesions with contact bleeding at vulva, vagina and cervix. Patients presenting with necrotizing vulvitis usually require radical vulvectomy [49]. It has been confirmed that the infection can be sexually transmitted [50] and therefore recommended that sexual partners of patients with genital amoebiasis should be examined and promptly treatment.

Most cases of the genital tract involving cervix, vulva and penis present as ulcerating mass sometimes clinically suspected to have tumor [51]. On careful assessment, a few of these cases have confirmed the co-existence of genital amoebiasis with carcinoma [52]. Though rare, there is need to emphasize for histopathological confirmation of all suspected genital malignancies due to the resemblance of genital amoebiasis to a carcinoma. The co-existence of amoebiasis with carcinoma is of interest and suggestive of etiological relationship that needs to be investigated. Cases of endometrial amoebiasis that were rare or uncommon are emerging in literature [53] and there is need for follow-up studies.

\subsection{Genital Leishmaniasis}

Leishmaniases are a group of diseases caused by protozoans of the genus Leishmania. The diseases affect 1-2 million people per year between latitudes $45^{\circ}$ North and $32^{\circ}$ South. Each species in the genus has an animal reservoir and can be transmitted to humans by sandfly bites. Following inoculation organisms are taken up by macrophages where they are able to resist degradation. The ensuing release of cytokines and cell mediated immune response to infection leads to the development of lesions. Cutaneous leishmaniasis is usually characterized by chronic localized ulceration developing at the site of inoculation. Genital lesions are rare but have been reported in South America [54, 55]. Vulval lesions have been reported in female patients [56]. Leishmania chagasi and L. donovani may cross the placenta and infect the foetus. Multiple case reports discuss infected women giving birth to infected infants, and to infants with disseminated disease. Untreated cases are linked with spontaneous pregnancy loss as well as active newborn disease [57]. Currently, diagnosis is by demonstrating the presence of Leishmania amastigotes in smear or biopsy material. It is possible that the use of culture and polymerase chain reaction (PCR) methods will increase detection rates of genital/congenital leishmaniasis in endemic regions. However, mild cases caused by certain species such as Leishmania peruviana that resolves spontaneously are likely to be missed.

\subsection{Malaria in Pregnancy}

Malaria is the most important parasitic infection in humans, transmitted by the bite of the sporozoite-bearing female anopheles mosquito. There are five types of malarial parasites, viz: Plasmodium falciparum, $P$ vivax, $P$ malariea, $P$ ovale and $P$. knowlesi. Worldwide, an estimated 300-500 million malaria cases occur annually, with approximately 1-3 million deaths per year. Populations at an increased risk for mortality include expectant mothers, young children aged 1-5 years. The effect of malaria infection results from systemic infection comparable to any severe febrile illness in pregnancy.

Plamodium species are intracellular parasites known to cause lyses of infected and uninfected red blood cells (RBCs). Infection is commonly associated with suppression of hematopoiesis and increased clearance of RBCs by the spleen which leads to anaemia in pregnant women and foetus. In the mother malaria leads to miscarriage, premature birth, intrauterine growth restriction (IUGR), still birth largely as result of maternal-fetal conflict [58]. Maternal-fetal conflict derives from the battle over scarce nutritional resources. The altered maternal-fetal relationship in the presence of an infectious disease has adverse outcomes on reproductive health $[59,60]$. 
The infection and aspects of the immune response contribute to poor pregnancy outcomes of prematurity and fetal intrauterine growth retardation. The effect on prematurity is not entirely clear, but women with an active infection and a fetus exposed to parasitized maternal erythrocytes may develop an immunologic response that contributes to stimulus of early onset of labour [61].

The effect of malaria on IUGR appears to be associated with the system of nutrient transport to the foetus [low supply of oxygen and nutrients]. The high-density and or prolonged parasite infection in placental blood and the consequent cellular immune response require substantial nutrients and thus leave less nutrients (glucose and oxygen) available for passage to the fetus. Further, erythrocytes infected with $P$. falciparum congregate in the maternal placental vascular space creating low pressure blood flow, and infected RBC adherence to endothelial cells allow parasites to sequester and replicate in placenta leading to miscarriage, still birth, abortion [62].

\section{Conclusion}

Parasitic protozoan infections categorised as neglected tropical diseases (NTDs), specifically trichomoniasis, amoebiasis, toxoplamosis, leishmaniasis and malaria directly or indirectly lead to a spectrum of adverse effects that impact negatively on human reproductive health. These infections are associated with increasing morbidity and mortality in both men and women of reproductive age worldwide. Therefore, more global attention to early diagnosis and treatment of these parasitic infections is warranted.

To bring infections to the limelight there is need to highlight the close relationship between these parasitic infections and contraception and integration of information on parasitic infections into reproductive tract infections (RTI) services (at family planning clinics). Though this may not reach other risk groups such as ado lescents, menopausal women and men, it is important that the information can easily be disseminated from this point. Screening offers the best method for identifying women and men with asymptomatic infections and early clinical manifestations that be treated early enough before irreversible organ/tissue destruction.

Lack of appropriate action and focus to date on the infections clearly illustrates the importance of new partnerships and new approaches to scaling up intervention strategies. To make progress in this area, there is need for joint action between maternal, sexual and reproductive health and neglected tropical disease communities. Health workers and communities need more information on the multiple impacts of these infections and how they can be diagnosed and promptly treated.

As health professionals, we should view reproductive health as encompassing sexual health, the purpose of which is the enhancement of life and personal relations, and not merely counseling and care related to reproduction and sexually transmitted diseases .

\section{Acknowledgements}

We acknowledge assistance of Mr. A. Wekesa of Egerton University, Kenya for review and editing services that enriched the manuscript.

\section{References}

[1] WHO, 2008

http://www.who.int/topics/reproductive_health/en/].

[2] Hall, Kelli Stidham, Caroline Moreau, and James Trussell. "Determinants Of And Disparities In Reproductive Health Service Use Among Adolescent And Young Adult Women In The United States, 2002-2008." American Journal of Public Health 102.2 (2012): 359-367.

[3] Freedman LP, Isaacs SL. Human rights and reproductive choice. Studies in Family Planning, 1993, 24:18-30. (UN Programme of Action adopted at the International Conference on Population and Development, Cairo, 5-13 September 1994.

[4] United Nations Population Fund (UNFPA), Male involvement in reproductive health, including family planning and sexual health, Technical Report, New York: UNFPA, 1995, No. 28.]

[5] Faundes A, Hardy E, Pinotti JA. Commentary on women's reproductive health: means or end? International Journal of Gynecology and Obstetrics. 1989, (supp) 3:115-8.] (UN Programme of Action adopted at the International Conference on Population and Development, Cairo, 5-13 September 1994.

[6] Carlton JM, Hirt RP, Silva JC, Delcher AL, Schatz M, Zhao Q, et al. Draft genome sequence of the sexually transmitted pathogen Trichomonas vaginalis. Science. 2007; 315:207-212.

[7] Fichorova RN, Trifonova RT, Gilbert RO, Costello CE, Hayes GR, Lucas JJ, et al. Trichomonas vaginalis lipophosphoglycan triggers a selective upregulation of cytokines by human female reproductive tract epithelial cells. Infect Immun. 2006; 74:5773-5779.

[8] Huang K-Y, Chien K-Y, Lin Y-C, Hsu W-M, Fong IK, Huang $\mathrm{P}-\mathrm{J}$, et al. A proteome reference map of Trichomonas vaginalis. Parasitol Res. 2009; 104:927-933.

[9] Singh BN, Hayes GR, Lucas JJ, Sommer U, Viseux N, Mirgorodskaya E, et al. Structural details and composition of Trichomonas vaginalis lipophosphoglycan in relevance to the epithelial immune function. Glycoconj J. 2009; 26:3-17.

[10] Zubácová Z, Cimburek Z, Tachezy J. Comparative analysis of trichomonad genome sizes and karyotypes. Mol Biochem Parasitol. 2008; 161:49-54.

[11] Alderete JF, Kasmala L, Metcalfe E, Garza GE. Phenotypic variation and diversity among Trichomonas vaginalis isolates and correlation of phenotype with trichomonal virulence determinants. Infect Immun. 1986; 53:285-293.

[12] McClelland RS. Trichomonas vaginalis infection: can we afford to do nothing? J Infect Dis.2008; 197:487-489.

[13] Shafir SC, Sorvillo FJ, Smith L. Current issues and considerations regarding trichomoniasis and human immunodeficiency virus in African-Americans. Clin Microbiol Rev. 2009; 22:37-45. 
[14] Smith LM, Wang M, Zangwill K, Yeh S. Trichomonas vaginalis infection in a premature newborn. J Perinatol. 2002; 22:502-503.

[15] Benchimol M, de Andrade Rosa I, da Silva Fontes R, Burla Dias AJ. Trichomonas adhere and phagocytose sperm cells: adhesion seems to be a prominent stage during interaction. Parasitol Res.2008; 102:597-604.

[16] Sena AC, Miller WC, Hobbs MM, Schwebke JR, Leone PA, Swygard $\mathrm{H}$, et al. Trichomonas vaginalis infection in male sexual partners: implications for diagnosis, treatment, and prevention. Clin Infect Dis. 2007; 44:13-22.

[17] Caliendo AM, Jordan JA, Green AM, Ingersoll J, Diclemente RJ, Wingood GM. Real-time PCR improves detection of Trichomonas vaginalis infection compared with culture using self-collected vaginal swabs. Infect Dis Obstet Gynecol. 2005; 13:145-150.

[18] Johnson LF, Coetzee DJ, Dorrington RE. Sentinel surveillance of sexually transmitted infections in South Africa: a review. Sex Transm Infect. 2005; 81:287-293.

[19] Yadav M, Gupta I, Malla N. Kinetics of immunoglobulin G, $\mathrm{M}, \mathrm{A}$ and $\mathrm{IgG}$ subclass responses in experimental intravaginal trichomoniasis: prominence of $\mathrm{IgG1}$ response. Parasite Immunology.2005; 27:461-467.

[20] Van Der Pol B, Williams JA, Orr DP, Batteiger BE, Fortenberry JD. Prevalence, incidence, natural history, and response to treatment of Trichomonas vaginalis infection among adolescent women. J Infect Dis. 2005; 192:2039-2044.

[21] Weinstock H, Berman S, Cates W., Jr Sexually transmitted diseases among American youth: incidence and prevalence estimates, 2000. Perspect Sex Reprod Health. 2004; 36:6-10.]

[22] Simhan HN, Anderson BL, Krohn MA, Heine RP, Martinez de Tejada B, Landers DV, et al. Host immune consequences of asymptomatic Trichomonas vaginalis infection in pregnancy. Am J Obstet Gynecol. 2007; 196(59):e51-e55.

[23] Al-Mohammed HI, Hussein EM. Comparison between secretory leukocytic protease inhibitor and reactive nitrogen intermediates levels in cervicovaginal secretions from symptomatic and asymptomatic trichomoniasis Egyptians patients. J Egypt Soc Parasitol. 2006; 36:763-777.

[24] Montoya JG, Liesenfeld O. Toxoplasmosis. Lancet. 2004; 363(9425):1965-76.

[25] Tenter AM, Heckeroth AR, Weiss LM. Toxoplasma gondii: from animals to humans. Int $J$ Parasitol. 2000; 30(1213):1217-58.

[26] Weiss LM, Dubey JP. Toxoplasmosis: A history of clinical observations. Int J Parasitol. 2009; 39(8):895-901.

[27] Hill D, Dubey JP. Toxoplasma gondii: transmission, diagnosis and prevention. Clin Microbiol Infect. 2002; 8(10):634-40.

[28] Sharifian M, Dalimi A, Kazemi B. Early diagnosis of toxoplasmosis by PCR method in the blood of experimentally infected rats. $J$ Vet Res.2003; 58(4):323-327.

[29] Zare F, Dalimi A, Ghaffarifar F. Detection of active Toxoplasma godii (RH strain) in the different body tissues of experimentally infected rats. Modares J Med Sci. 2006; 9(1):19-23.
[30] Martinez-Garcia F, Regadera J, Mayer R, Sanchez S, Nistal M. Protozoan infections in the male genital tract. $J$ Urol. 1996; 156 (2 Pt 1):340-9.

[31] Nistal M, Santana A, Paniaqua R, Palacios J. Testicular toxoplasmosis in two men with the acquired immunodeficiency syndrome (AIDS). Arch Pathol Lab Med. 1986; 110(8):744-6.

[32] Barreto F, Hering F, Dall'oglio MF, Martini Filho D, Campagnari JC, Srougi M. Testicular toxoplasmosis: a rare case of a testicular mass. Actas Urol Esp. 2008; 32(6):666-8.

[33] De Paepe ME, Guerrieri C, Waxman M. Opportunistic infections of the testis in the acquired immunodeficiency syndrome. Mt Sinai J Med. 1990; 57(1):25-9.

[34] Crider SR, Horstman WG, Massey GS. Toxoplasma orchitis: report of a case and a review of the literature. Am J Med. 1988; 85(3):421-4.

[35] Haskell L, Fusco MJ, Ares L, Sublay B. Disseminated toxoplasmosis presenting as symptomatic orchitis and nephrotic syndrome. Am J Med Sci. 1989; 298(3):185-90.

[36] Suresh Babu PS, Nagendra K, Navaz RS, Ravindranath HM. Congenital toxoplasmosis presenting as hypogonadotropic hypogonadism. Indian J Pediatr. 2007; 74(6):577-9.

[37] Zhou YH, Lu YJ, Wang RB, Song LM, Shi F, Gao QF, et al. Survey of infection of Toxoplasma gondii in infertile couples in Suzhou countryside]. Zhonghua Nan Ke Xue. 2002; 8(5): 350-2.

[38] Remington JS, Desmonts G. Toxoplasmosis. In: Remington JS, Klein JO, editors. Infectious diseases of the fetus and newborn infant. 3. Philadelphia: WB Saunders; 1990. pp. 89-195.

[39] Ebbesen P. Placenta physiology. In: Ambroise-Thomas P, Petersen E, editors. Congenital toxoplasmosis: scientific background, clinical management and control. Paris: Springer-Verlag; 2000. pp. 27-35.

[40] Remington JS, McLeod R, Desmonts G. Toxoplasmosis. In: Remington JS, Klein JO, editors. Infectious diseases of the fetus and newborn infant. 4. Philadelphia: WB Saunders; 1995. pp. 140-267.

[41] Chatterton JMW. Pregnancy. In: Ho-Yen DO, Joss AWL, editors. Human toxoplasmosis. Oxford: Oxford University Press; 1992. pp. 144-83.

[42] Hayde M, Pollak A. Clinical picture: neonatal signs and symptoms. In: Ambroise-Thomas P, Petersen E, editors.Congenital toxoplasmosis: scientific background, clinical management and control. Paris: Springer-Verlag France; 2000. pp. 153-64.

[43] Mandell GE, Bennett JE, Dolin R; Principles and Practice of Infectious Diseases. 5th edition,Philadelphia: Churchill Livingstone, 2000.

[44] Antony SJ, Lopez-Po P. Genital amebiasis: historical perspective of an unusual disease presentation.Urology1999; $54: 952-5$.

[45] Mhlanga BR, Lanoie LO, Norris HJ, et al. Amebiasis complicating carcinomas: a diagnostic dilemma. Am J Trop Med Hyg 1992; 46:759-64.

[46] Othman NH, Ismail AN. Endometrial amoebiasis. Eur $J$ Obstet Gynecol Reprod Biol 1993; 52:135-7. 
[47] Calore EE, Calore NMP, Cavaliere MJ. Salpingitis due to Entamoeba histolytica. Braz J Infect Dis2002; 6:97-9.

[48] Citronberg RJ, Semel JD. Severe vaginal infection with Entamoeba histolytica in a woman who recently returned from Mexico: case report and review. Clin Infect Dis 1995; 20:700715.

[49] Mylius RE, Ten Seldam RE. Venereal infection by Entamoeba histolytica in a New Guinea couple. Tropical and Geographical Medicine 1962; 14:20-6.

[50] Nopdonrattakoon L Amoebiasis of the female genital tract: a case report. J Obstet Gynaecol Res. 1996 Jun; 22(3):235-8.

[51] A.J. Veliath R. Bansal V. Sankaran, P. Rajaram, S. Parkash. Genital amebiasis . Internationa journal of Gynecology and Obstetrics June 1987 Volume 25, Issue 3, Pages 249-256 DOI: http://dx.doi.org/10.1016/0020-7292(87)90242-6.

[52] Gupta Shipra, Nivedita Sarda, Seema Singhal, Anil Jain, Renu Arora .Endometrial Amoebiasis: A Rare Cause of Postmenopausal Vaginal Discharge. Sch J Med Case Rep 2014; 2(1):38-39.

[53] Cabello I, Caraballo A, Millan Y. Leishmaniasis in the genital area. Rev Inst Med Trop Sao Paolo2002; 44:105-7.

[54] Castro-Coto A, Hidalgo-Hidalgo $\mathrm{H}$, Solano-Aguilar E, et al. Leishmaniasis en organos genitales. Med Cutan Ibero Lat Am 1987; 15:145-50.
[55] Blickstein I, Dgani R, Lifschitz-Mercer B. Cutaneous leishmaniasis of the vulva. Int J Gunaecol Obstet 1993; 42:46-7.

[56] Figueiro' -Filho, E. A., Duarte, G. \& El-Beitune, P., et al. (2004). Visceral Leishmaniasis (kala-azar) and Pregnancy. Infect Dis Obstet Gynecol, 12(1), 31-40.

[57] Abrams ET, Meshnick SR. Abrams ET, Meshnick SR. Malaria during pregnancy in endemic areas: a lens for examining maternal-fetal conflict. Am J Hum Biol. 2009 Sep-Oct; 21(5):643-50. doi: 10.1002/ajhb.20919.

[58] Breymann C, Iron Deficiency and Anaemia in Pregnancy: Modern Aspects of Diagnosis and Therapy. Blood Cells, Molecules, and Diseases. 2002; 29(3) Nov/Dec: 506-516.

[59] Ezechi Oliver and Kalejaiye Olufunto (2012). Management of Anaemia in Pregnancy, Anemia, Dr. Donald Silverberg (Ed.), ISBN: 978-953-51-0138-3, InTech, Available from: http://www.intechopen.com/books/anemia/management-ofanaemia-in-pregnancy;

[60] K. Marsh and R. W. Snow, "Malaria transmission and morbidity," Parassitologia, vol. 41, no. 1-3, pp. 241-246, 1999.

[61] Nicaise Tuikue Ndam and Philippe Deloron. Molecular Aspects of Plasmodium falciparum Infection during Pregnancy. Journal of Biomedicine and Biotechnology Volume 2007, Article ID 43785, 13 pages doi:10.1155/2007/43785. 